\title{
Metal/ SrTiO3 two-dimensional electron gases for spin-to-charge conversion
}

Vicente-Arche, Luis M.; Mallik, Srijani; Cosset-Cheneau, Maxen; Noël, Paul; Vaz, Diogo C.; Trier, Felix; Gosavi, Tanay A.; Lin, Chia Ching; Nikonov, Dmitri E.; Young, lan A.

Total number of authors:

15

Published in:

Physical Review Materials

Link to article, DOI:

10.1103/PhysRevMaterials.5.064005

Publication date:

2021

Document Version

Publisher's PDF, also known as Version of record

Link back to DTU Orbit

Citation (APA):

Vicente-Arche, L. M., Mallik, S., Cosset-Cheneau, M., Noël, P., Vaz, D. C., Trier, F., Gosavi, T. A., Lin, C. C., Nikonov, D. E., Young, I. A., Sander, A., Barthélémy, A., Attané, J. P., Vila, L., \& Bibes, M. (2021). Metal/ $\mathrm{SrTiO}_{3}$ two-dimensional electron gases for spin-to-charge conversion. Physical Review Materials, 5(6), [064005]. https://doi.org/10.1103/PhysRevMaterials.5.064005

\section{General rights}

Copyright and moral rights for the publications made accessible in the public portal are retained by the authors and/or other copyright owners and it is a condition of accessing publications that users recognise and abide by the legal requirements associated with these rights.

- Users may download and print one copy of any publication from the public portal for the purpose of private study or research.

- You may not further distribute the material or use it for any profit-making activity or commercial gain

- You may freely distribute the URL identifying the publication in the public portal 


\title{
Metal/SrTiO 3 two-dimensional electron gases for spin-to-charge conversion
}

\author{
Luis M. Vicente-Arche, ${ }^{1, *}$ Srijani Mallik, ${ }^{1, *}$ Maxen Cosset-Cheneau, ${ }^{2}$ Paul Noël, ${ }^{2, \dagger}$ Diogo C. Vaz, ${ }^{1, \star}$ Felix Trier $\odot,{ }^{1, \S}$ \\ Tanay A. Gosavi, ${ }^{3}$ Chia-Ching Lin, ${ }^{3}$ Dmitri E. Nikonov, ${ }^{3}$ Ian A. Young, ${ }^{3}$ Anke Sander $\odot,{ }^{1}$ Agnès Barthélémy, ${ }^{1}$ \\ Jean-Philippe Attané, ${ }^{2}$ Laurent Vila $\odot,^{2}$ and Manuel Bibes ${ }^{1}{ }^{1}$ \\ ${ }^{1}$ Unité Mixte de Physique, CNRS, Thales, Université Paris-Saclay, 91767 Palaiseau, France \\ ${ }^{2}$ Université Grenoble Alpes, CEA, CNRS, INP, IRIG-Spintec, Grenoble, France \\ ${ }^{3}$ Components Research, Intel Corporation, Hillsboro, Oregon 97124, USA
}

(Received 9 December 2020; accepted 3 May 2021; published 15 June 2021)

\begin{abstract}
$\mathrm{SrTiO}_{3}$-based two-dimensional electron gases (2DEGs) can be formed through the deposition of epitaxial oxides like $\mathrm{LaAlO}_{3}$ or of reactive metals such as Al. Such 2DEGs possess a finite Rashba spin-orbit coupling that has recently been harnessed to interconvert charge and spin currents through the direct and inverse Edelstein and spin Hall effects. Here we compare the formation and properties of 2DEGs generated in $\mathrm{SrTiO}_{3}$ by the growth of Al, Ta, and Y ultrathin films by magnetron sputtering. By combining in situ and ex situ x-ray photoelectron spectroscopy (XPS) we gain insight into the reduction of the $\mathrm{SrTiO}_{3}$ and the appearance of $\mathrm{Ti}^{3+}$ states associated with 2DEG formation, its reoxidation by exposure to the air, and the transformation of the metal into its binary oxides. We extract the carrier densities through magnetotransport and compare them with the XPS data. Finally, working with samples covered by an extra layer of $\mathrm{NiFe}$, we perform spin-pumping ferromagnetic resonance experiments and investigate spin-charge conversion as a function of gate voltage. We identify trends in the data across the different sample systems and discuss them as a function of the carrier density and the transparency of the metal oxide tunnel barrier.
\end{abstract}

DOI: 10.1103/PhysRevMaterials.5.064005

\section{INTRODUCTION}

Since their discovery in 2004 [1], two-dimensional electron gases (2DEGs) based on $\mathrm{SrTiO}_{3}$ (STO) have been shown to possess a wide array of fascinating properties [2,3]. They are superconducting below about $200 \mathrm{mK}$ [4], their transport response is very sensitive to gate voltages [5], they harbor a sizeable Rashba spin-orbit coupling (R-SOC) [6,7] and even signs of magnetic order [8]. While these discoveries challenge our understanding of quantum matter, they also trigger some interest for technological applications. Indeed, STO 2DEGs have been functionalized as transistors into integrated circuits [9], as rewritable photodetectors [10] or, more recently, as the read-out unit [11] of future spin-based logic devices coined MESO (for magnetoelectric spin orbit) proposed by Intel Corporation [12]. In this latter example, the R-SOC of the 2DEG is harnessed to efficiently convert spin currents into charge currents in a bipolar way, through the inverse Edelstein effect [13]. This spin-charge conversion was originally demonstrated in STO 2DEGs classically formed by the epitaxial growth of $\mathrm{LaAlO}_{3}$ at high temperature $[14,15]$,

\footnotetext{
*These authors contributed equally to this work.

${ }^{\dagger}$ Present address: Department of Materials, ETH Zürich, Hönggerbergring 64, 8093 Zürich, Switzerland.

Present address: CIC nanoGUNE BRTA, Tolosa Hiribidea, 76, 20018 Donostia, San Sebastian, Spain.

§Present address: Department of Energy Conservation and Storage, Technical University of Denmark, 2800 Kgs. Lyngby, Denmark.
}

but later was also reported in STO 2DEGs generated by the simple deposition of $\mathrm{Al}$ at room temperature $[11,16,17]$. This simpler and more integrable synthesis process, combined with the larger conversion efficiency and its survival at room temperature are very appealing for applications and prompted us to investigate metal/STO 2DEGs in greater detail.

In this paper, we report the synthesis of 2DEGs in STO by depositing various reactive metals by magneton sputtering [18]. We selected $\mathrm{Al}, \mathrm{Ta}$, and $\mathrm{Y}$, which can all be grown as smooth ultrathin films and whose oxide formation enthalpies satisfy the thermodynamic criteria to reduce the $\mathrm{SrTiO}_{3}\left(\Delta H_{\mathrm{f}, \mathrm{ox}}<-250 \mathrm{~kJ} / \mathrm{mol} \mathrm{O}\right)$ [19-21]. Using an $\mathrm{x}-$ ray photoelectron spectroscopy (XPS) chamber, connected in ultrahigh vacuum (UHV) to our sputtering chamber, we could monitor the formation of the 2DEG through the appearance of $\mathrm{Ti}^{3+}$ states, as well as its degradation after the samples were exposed to the air. We confirmed the presence of the $2 \mathrm{DEG}$ through temperature dependence resistance measurements and Hall experiments from which we deduced the carrier density in a two-band model. We found that both the $\mathrm{Ti}^{3+}$ concentration and the carrier density tend to increase with the metal thickness deposited, with subtle differences between the three systems. Finally, we prepared samples in which the reactive metal was covered by a $\mathrm{Ni}_{80} \mathrm{Fe}_{20}$ (permalloy, NiFe) layer so as to perform spinpumping experiments by ferromagnetic resonance (SP-FMR). We measured the charge current generated transverse to the injected spin current due to the inverse Edelstein effect as a function of the gate voltage for samples based on $\mathrm{Al}$, 
Ta, and Y, having different ungated carrier density values. We finally compared the gate dependencies across various sample types to draw conclusions on the role of the complex multiorbital band structure on the spin-charge conversion process.

\section{EXPERIMENTAL DETAILS}

Preparation of STO substrates. Single-crystal STO (001) substrates $(5 \mathrm{~mm} \times 5 \mathrm{~mm} \times 0.5 \mathrm{~mm}$, one-side polished with miscut angles $<0.1^{\circ}$ ) were purchased from SurfaceNet $\mathrm{GmbH}$. The as-received substrates, with a mixed surface termination $\left(\mathrm{SrO}-\mathrm{TiO}_{2}\right)$, were cleaned by sonicating in deionized water, acetone, and isopropanol and subsequently dried with nitrogen. This process was repeated until the cleanliness of the surface was confirmed by AFM. The cleaned substrates were inserted in a UHV system for metal deposition by magnetron sputtering and in situ XPS measurements.

Metal deposition. The metal deposition was performed in a commercial dc magnetron sputtering system PLASSYS MP450S with a base pressure of $9 \times 10^{-8}$ mbar. The different deposition rates were derived by means of $\mathrm{x}$-ray reflectometry (XRR) on thicker samples grown under the same conditions. All metal depositions were carried out on different STO substrates at room temperature. The Ar gas flow and the current intensity were fixed to 5.2 SCCM (SCCM denotes cubic centimeter per minute at STP) and $30 \mathrm{~mA}$, respectively. The pressure of the chamber during depositions was $5.3 \pm 0.2 \times$ $10^{-4}$ mbar. The plasma power varied slightly depending on which metal was being deposited: $10 \mathrm{~W}$ for $\mathrm{Al}, 9 \mathrm{~W}$ for $\mathrm{Ta}$, and $8 \mathrm{~W}$ for $\mathrm{Y}$. The NiFe was also deposited by dc magnetron sputtering, and capped with $1.5 \mathrm{~nm}$ of $\mathrm{Al}$, that transformed into $\mathrm{AlO}_{\mathrm{x}}$ after exposure to air.

XPS measurements. The XPS measurements were performed at room temperature using an Omicron NanoTechnology $\mathrm{GmbH}$ system with a base pressure of $5 \times 10^{-10} \mathrm{mbar}$, using a $\mathrm{Mg} K_{\alpha}$ source $(h v=1253.6 \mathrm{eV})$ operating at $20 \mathrm{~mA}$ and $15 \mathrm{kV}$. The spectra were obtained at a pass energy of $20 \mathrm{eV}$. In situ XPS measurements were performed before and immediately after the deposition of $\mathrm{Al}$, Ta, or $\mathrm{Y}$ on different samples. Ex situ XPS measurements were also carried out on the same samples after they were exposed to air. The fitting of the spectra was carried out using CASAXPS (CasaSoftware Ltd.).

Magnetotransport properties measurements. The samples were measured with a Dynacool system from Quantum Design after bonding with Al wires in van der Pauw configuration. During the transport measurements of $\mathrm{AlO}_{\mathrm{x}} / \mathrm{NiFe} / \mathrm{metal} / \mathrm{STO}$ samples, the $\mathrm{NiFe}$ and $2 \mathrm{DEG}$ signal were probed in parallel [22]. Both contributions were disentangled considering the following: for the longitudinal configuration $R_{x x}$, two resistances in parallel were measured so that the resistance of the $2 \mathrm{DEG}$ alone is given by

$$
R_{2 \mathrm{DEG}}=\left(R_{M} \times R_{\mathrm{Total}} /\left(R_{M}-R_{\text {Total }}\right) .\right.
$$

For the transverse configuration $R_{x y}$ (Hall resistance), besides $R_{M}$ and $R_{2 \mathrm{DEG}}$ the Hall voltages generated in each layer must be also considered. These circuits can be simplified using Millman's theorem [23] so that the Hall resistance of the 2DEG alone $R_{H}$, 2DEG is given by

$$
\begin{aligned}
R_{H, 2 \mathrm{DEG}}= & R_{H, \text { Total }} \times\left[\left(R_{M} / R_{\text {Total }}\right)+1\right]^{2} \\
& -R_{H, M} \times\left(R_{2 \mathrm{DEG}} / R_{M}\right)^{2} .
\end{aligned}
$$

The 2DEG contribution was then fitted with a standard two-band model in order to extract carrier densities and mobilities.

Spin pumping. The spin-pumping experiments were carried out using a Bruker ESP300E X-band CW spectrometer at $9.68 \mathrm{GHz}$, with a loop-gap Bruker ER 4118X-MS5 cavity, and using a microwave power of $5 \mathrm{~mW}$ or less to remain in the linear regime and avoid thermal effects [24]. The DC voltage generated transverse to the magnetization was measured using a Keithley $2182 \mathrm{~A}$ nanovoltmeter. The gate voltage was applied using a Keithley 2400 source meter. The sample was initialized by sweeping the back-gate voltage from +200 to $-200 \mathrm{~V}$, and then back to $+200 \mathrm{~V}$, to avoid any hysteretic behavior. The measurement was then performed for different gate voltages, from +200 to $-200 \mathrm{~V}$. The measured signals were observed to be linear with rf power up to $5 \mathrm{~mW}$ with no indications of heating [24].

\section{RESULTS}

\section{A. XPS}

Prior to any deposition, XPS was performed individually on all bare STO substrates used to prepare samples for this work. All showed similar spectra for the Ti $2 p$ core levels, an example of which is shown in the inset of Fig. 1(a). During the fitting, adventitious carbon was used as a charge reference to obtain the $\mathrm{Ti}^{4+} 2 p_{3 / 2}$ peak position $\sim 458.6 \mathrm{eV}$ that is consistent with previously reported values for STO [25-28]. The fits of the spectra suggest the presence of $\mathrm{Ti}^{4+}$ only, as expected for as-received insulating STO single crystals.

The Ti $2 p$ core-level spectra, collected in situ after depositing different metal thicknesses, are shown in Fig. 1 for $\mathrm{Al}$ [Figs. 1(a)-1(c)], Ta [Figs. 1(d)-1(f)], and Y [Figs. 1(g)-1(i)]. Signs of the reduction of STO are visible from the spectra as the lower binding energy peaks of $\mathrm{Ti}^{4+}\left(\mathrm{Ti}^{3+}\right.$ and $\left.\mathrm{Ti}^{2+}\right)$ appear upon deposition of the metal. Further, the $\mathrm{Ti}^{3+}$ and $\mathrm{Ti}^{2+}$ peak areas become larger with increasing metal thicknesses.

The Ti $2 p$ core-level spectra collected after exposing the same samples to air are shown in Fig. 2 for $\mathrm{Al}$ [Figs. 2(a)-2(c)], Ta [Figs. 2(d)-2(f)], and Y [Figs. 2(g)-2(i)].

For the Ta case, the Ti $2 p$ core-level energy region overlaps with the energy range of Ta $4 p_{1 / 2}$. Thus, it was necessary to consider its contribution to determine the Ti valence states. In this case, we analyzed a wider energy region which included the Ta $4 p_{3 / 2}$ core level in order to constrain the contribution of Ta $4 p_{1 / 2}$ during the analysis.

The contribution of the reduced titanium was determined by means of an active fitting process during which all possible valence states were considered. The peak area ratio $\mathrm{Ti}$ $2 p_{1 / 2}:$ Ti $2 p_{3 / 2}$ was fixed to 0.5 and fitted with a GaussianLorentzian (70:30) peak line shape. Finally, we achieved the best agreement for all the spectra when the constraints were applied as in Table I.

After the analysis, we confirmed that titanium reduces differently depending on the metal deposited. For a better 


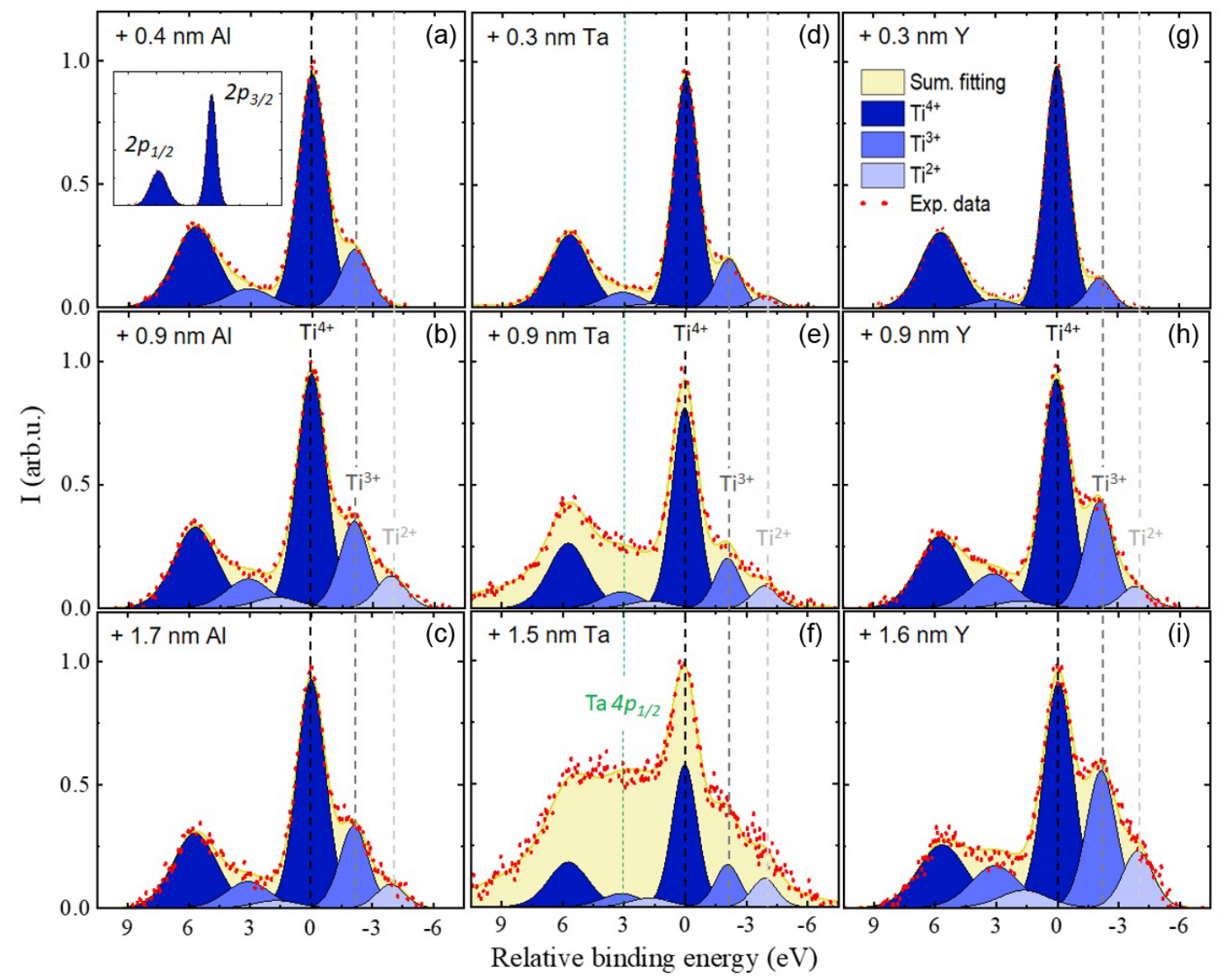

FIG. 1. Ti $2 p$ core-level spectra obtained in situ when different thicknesses of: aluminium (a)-(c), tantalum (d)-(f), and yttrium (g)-(i) are deposited on STO. Ti $2 p$ spectra for the bare STO substrate is shown as an inset in (a). All the spectra have been normalized to the maximum intensity and the binding energy values are relative to the $\mathrm{Ti}^{4+} 2 p_{3 / 2}$ peak position. The color code is the same for all figures and it is shown in (g). Note that, for the Ta case, the Ti $2 p$ core-level energy region overlaps with the energy range of Ta $4 p_{1 / 2}$. Therefore, for better visualization the fitted Ta peaks have been removed from the spectra.

visualization, the evolution of Ti valence states is plotted in Fig. 3 as a function of the metal thicknesses for all the samples measured in situ (open circles) as well as after being exposed to air (filled circles). Comparing the relative concentration of $\mathrm{Ti}^{3+}$ present in the STO substrates before exposing the samples to air (open circles), it can be inferred that most of the reduction of STO occurs for the first few angstroms deposited $(0.5 \mathrm{~nm})$ and is highest for $\mathrm{Y}$, then for $\mathrm{Al}$ and for Ta.

TABLE I. Fitting parameters used for the XPS Ti $2 p$ spectra displayed in Figs. 1 and 2. FWHM stands for full width at half maximum and B.E. is for binding energy.

\begin{tabular}{ccccc}
\hline \hline & Peak & $\Delta \mathrm{E}\left(2 \mathrm{p}_{1 / 2}-2 \mathrm{p}_{3 / 2}\right)$ & FWHM & B.E. \\
\hline $\mathrm{a}$ & $\mathrm{Ti}^{4+} 2 p_{1 / 2}$ & $5.7 \mathrm{eV}$ & $2.5 \pm 0.1 \mathrm{eV}$ & \\
$\mathrm{b}$ & $\mathrm{Ti}^{4+} 2 p_{3 / 2}$ & & $1.7 \pm 0.1 \mathrm{eV}$ & $458.6 \mathrm{eV}$ \\
$\mathrm{c}$ & $\mathrm{Ti}^{3+} 2 p_{1 / 2}$ & $5.2 \mathrm{eV}$ & $\mathrm{a}^{*} 1$ & \\
$\mathrm{~d}$ & $\mathrm{Ti}^{3+} 2 p_{3 / 2}$ & & $\mathrm{~b}^{*} 1$ & $456.5 \mathrm{eV}$ \\
$\mathrm{e}$ & $\mathrm{Ti}^{2+} 2 p_{1 / 2}$ & $5.6 \mathrm{eV}$ & $\mathrm{a}^{* 1}$ & \\
$\mathrm{f}$ & $\mathrm{Ti}^{2+} 2 p_{3 / 2}$ & & $\mathrm{~b}^{* 1}$ & $454.7 \mathrm{eV}$ \\
\hline \hline
\end{tabular}

Furthermore, Fig. 3 also reflects the critical thickness needed to preserve the reduced $\mathrm{Ti}$ from reoxidation by the air. This critical thickness can be defined as the point where the in situ and ex situ curves for the $\mathrm{Ti}^{4+}$ valence state meet. The two data sets meet around 1.3-1.7 nm for $\mathrm{Al}, 1-1.3 \mathrm{~nm}$ for Ta, and beyond 1.6 for $\mathrm{nm}$ for $\mathrm{Y}$. This observation can be well correlated with the oxygen diffusion coefficients of $\mathrm{Y}_{2} \mathrm{O}_{3}, \mathrm{Ta}_{2} \mathrm{O}_{5}$, and $\mathrm{Al}_{2} \mathrm{O}_{3}$. It is known that the oxygen diffusion coefficient for $\mathrm{Ta}_{2} \mathrm{O}_{5}$ and $\mathrm{Al}_{2} \mathrm{O}_{3}$ are of similar order of magnitude whereas it is higher for $\mathrm{Y}_{2} \mathrm{O}_{3}$ [29]. Therefore, even if $\mathrm{Y}$ shows the highest reduction of STO, it is not a good candidate to protect the reduced metal/STO interface (and thus the 2DEG) in comparison with $\mathrm{Al}$ and $\mathrm{Ta}$. From these data we also determine the thickness of the oxide formed by the reduction of the STO upon metal deposition, that corresponds to the point where the concentration of $\mathrm{Ti}^{3+}$ becomes constant, i.e., $\sim 0.9 \mathrm{~nm}$ for $\mathrm{Al}, \sim 0.3$ for $\mathrm{Ta}$, and $\sim 1.2$ $\mathrm{nm}$ for Y. Ideally, for each system the sum of these two critical thicknesses indicates the deposited metal thickness above which the presence of nonoxidized metallic regions may be expected. This thickness is thus around $2.6 \mathrm{~nm}$ for Al, $1.6 \mathrm{~nm}$ for $\mathrm{Ta}$, and $2.8 \mathrm{~nm}$ for $\mathrm{Y}$. 


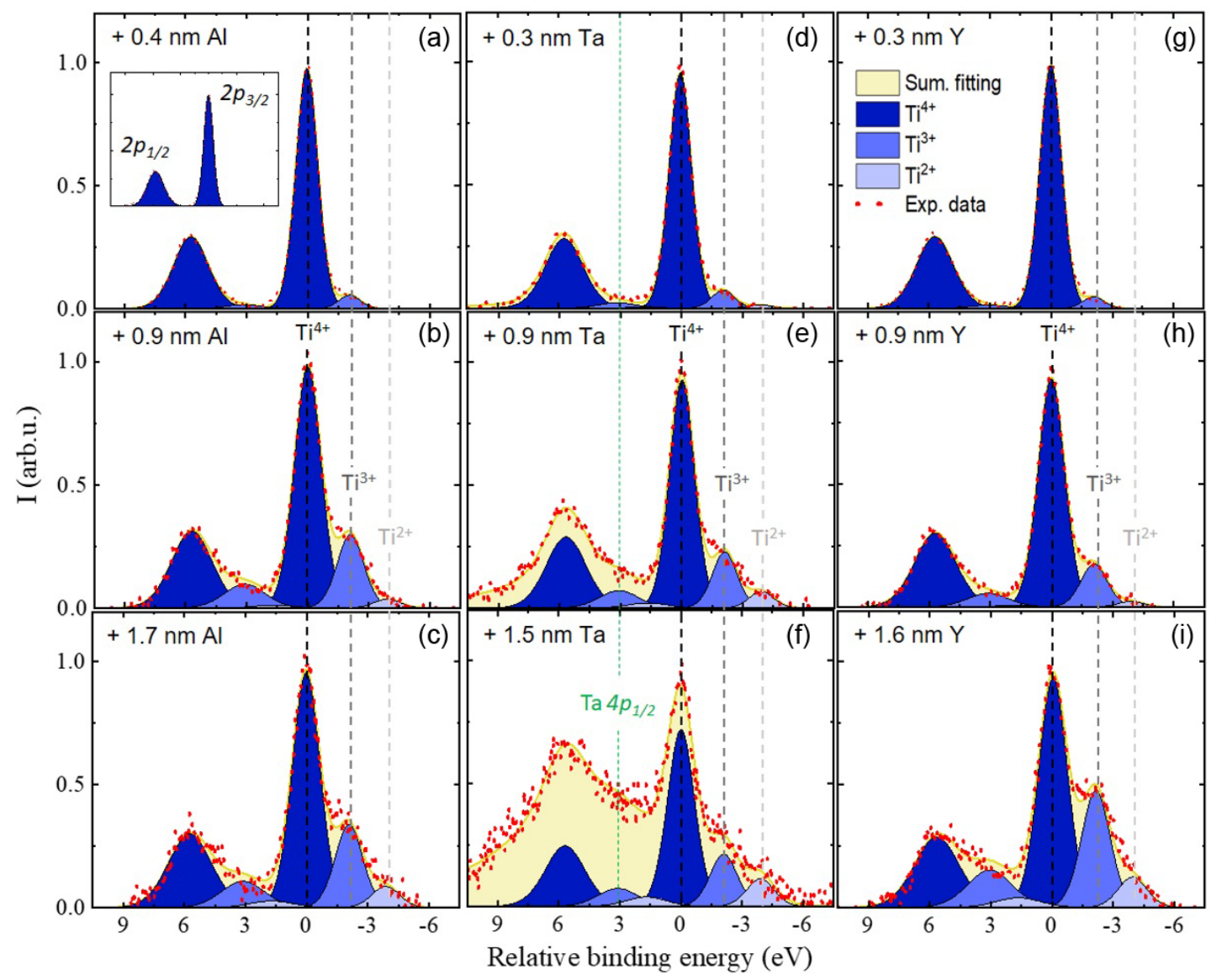

FIG. 2. Ti $2 p$ core-level spectra obtained $e x$ situ when different thicknesses of aluminium (a)-(c), tantalum (d)-(f), and yttrium (g)-(i) are deposited on STO. All the spectra have been normalized to the maximum intensity and the binding energy values are relative to the $T i^{4+} 2 p_{3 / 2}$ peak position. The color code is the same for all figures and it is shown in $(\mathrm{g})$.

In addition, we also studied the evolution of the metal valence states after each deposition. For the first depositions $(\sim 1 \mathrm{~nm})$, it is clearly visible that the metal gets oxidized at the STO interface. Further, by increasing the metal thickness we observe the contribution of nonoxidized metal along with its oxide states. As an example, Fig. 4 shows ex situ spectra having both nonoxidized metal and corresponding metal oxides contributions for specific thicknesses of the three metals.
The relative fraction of oxides and nonoxidized metal contributions obtained ex situ for different materials are shown in Fig. 5 as a function of metal thicknesses. By increasing the metal thickness, we observe the nonoxidized metal contribution for $\mathrm{Al}$ and $\mathrm{Y}$ along with their corresponding oxide valence state $\left(\mathrm{Al}^{3+}, \mathrm{Y}^{3+}\right)$. In contrast, in the case of Ta, first we observe the appearance of reduced valence states of Ta related to the formation of intermediate oxides $\left(\mathrm{Ta}^{4+}, \mathrm{Ta}^{2+}\right.$, $\mathrm{Ta}^{1+}$ ) which exhibit maxima depending on the thickness of
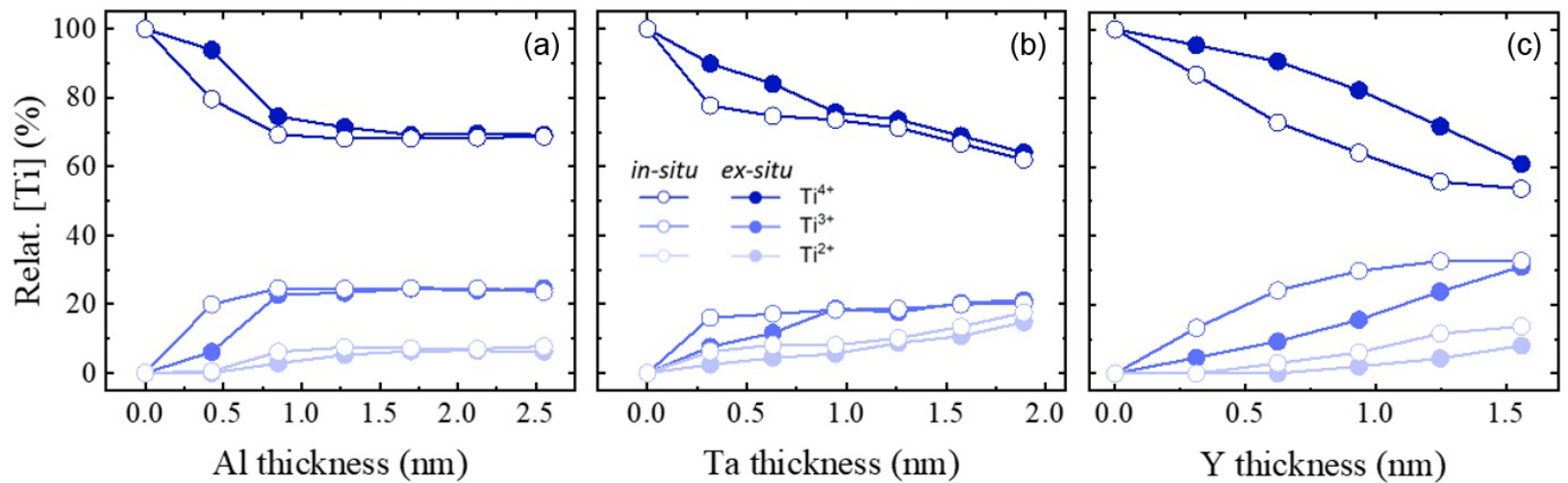

FIG. 3. Ti valence states evolution when increasing the thickness of $\mathrm{Al}$ (a), Ta (b), and Y (c). The open circles correspond to the spectra collected in situ. The filled circles correspond to the spectra collected for the same samples after being exposed to air. 

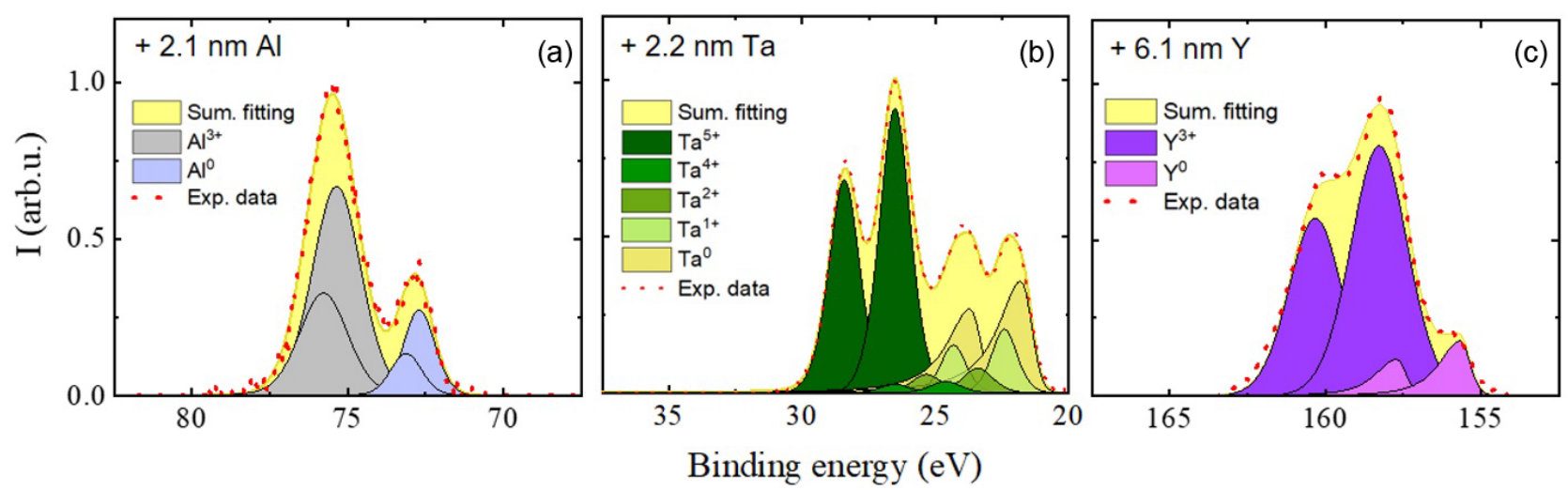

FIG. 4. Ex situ XPS spectra obtained after the deposition of $2.1 \mathrm{~nm}$ of aluminium (a), $2.2 \mathrm{~nm}$ of tantalum (b), and $6.1 \mathrm{~nm}$ of yttrium (c). It shows that aluminium and yttrium are mostly oxidized with a valence state $3+$ which could correspond to $\mathrm{Al}_{2} \mathrm{O}_{3}$ and $\mathrm{Y}_{2} \mathrm{O}_{3}$ respectively, whereas in the case of tantalum oxide several valence states $(1+, 2+, 4+, 5+)$ are present.

the deposited Ta layer. By further increasing the thickness we observe the contribution from metallic Ta. It should be noted that the appearance of a nonoxidized metal is observed in ex situ XPS spectra above a minimum thickness of the metal layers. Ideally, this contribution appears when no further oxidation of the metal is possible between the oxide layers formed by the oxidation from STO and from the air.

It can be observed in Fig. 5 that the minimum thickness at which the nonoxidized metal appears is similar for the $\mathrm{Al}$ and Ta cases $(\sim 1.5 \mathrm{~nm})$, while in the case of $\mathrm{Y}$ it is notably higher $(\sim 5 \mathrm{~nm})$. This can be explained by considering two phenomena happening simultaneously. The first is the stronger oxidation of Y upon reducing STO as observed in Fig. 3. The second is the higher oxygen diffusion coefficient [29-31] and thicker metal oxide layer thickness for $\mathrm{Y}$ when exposed to air. This is also confirmed by the metal oxide layer thicknesses $\left(t_{\mathrm{ox}}\right)$ obtained by means of XRR on thicker samples which are $t_{\mathrm{ox}, \mathrm{Y}_{2} \mathrm{O}_{3}} \approx 3.8 \mathrm{~nm}, t_{\mathrm{ox}, \mathrm{Al}_{2} \mathrm{O}_{3}} \approx 1.8 \mathrm{~nm}, t_{\mathrm{ox}, \mathrm{Ta}_{2} \mathrm{O}_{5}} \approx$ $1.6 \mathrm{~nm}$. Further, by increasing the thickness of the metal up to $\sim 60 \mathrm{~nm}$, the trends evolve towards the saturation of contributions from the nonoxidized metal and oxides where only the native oxide and nonoxidized metal underneath are detected (as shown by the crossed circle in Fig. 5). Finally, we note that for the $\mathrm{Al}$ and $\mathrm{Y}$ cases, the metallic contribution is detected in XPS at different thicknesses from those estimated from Fig. 3. For the Al case, this metallic contribution is detected before reaching the critical thickness of $2.6 \mathrm{~nm}$ of $\mathrm{Al}$ and may be explained by considering the relatively higher roughness of Al-based samples. Thus, it is likely that after depositing a nominal thickness of $1.7 \mathrm{~nm}$ of $\mathrm{Al}$ some regions are thicker than $2.6 \mathrm{~nm}$, favoring the presence of a metallic phase underneath. On the other hand, for the $\mathrm{Y}$ case, the thickness above which this metallic contribution is detected is much larger $(5.1 \mathrm{~nm})$ than the one estimated from Fig. 3 $(2.9 \mathrm{~nm})$. This difference is possibly due to an underestimated Y thickness oxidized by STO (1.2 nm), due to the insufficient probing depth of XPS to probe the whole 2DEG thickness.

\section{B. Transport measurements in metal//STO}

In Fig. 6 we show $R_{\mathrm{S}}$ vs T plots and the sheet resistance at $2 \mathrm{~K}$ of samples with different metal thicknesses. We observe a variation of $R_{\mathrm{S}}$ by one order of magnitude, which cannot be ascribed to the metallic capping thus implying the existence of an additional path for conduction related to the formation of a 2DEG when the STO is reduced, consistent with the XPS data. Figures 6(d)-6(f) show the thickness dependence of sheet resistance $\left(R_{\mathrm{S}}\right)$ at $2 \mathrm{~K}$ for $\mathrm{Al}$, Ta, and $\mathrm{Y}$, respectively. The sheet resistance increases with decreasing metal thickness and the samples become insulating below $\sim 1 \mathrm{~nm}$ as the oxide layer is not enough to protect the $2 \mathrm{DEG}$ region from reoxidation.
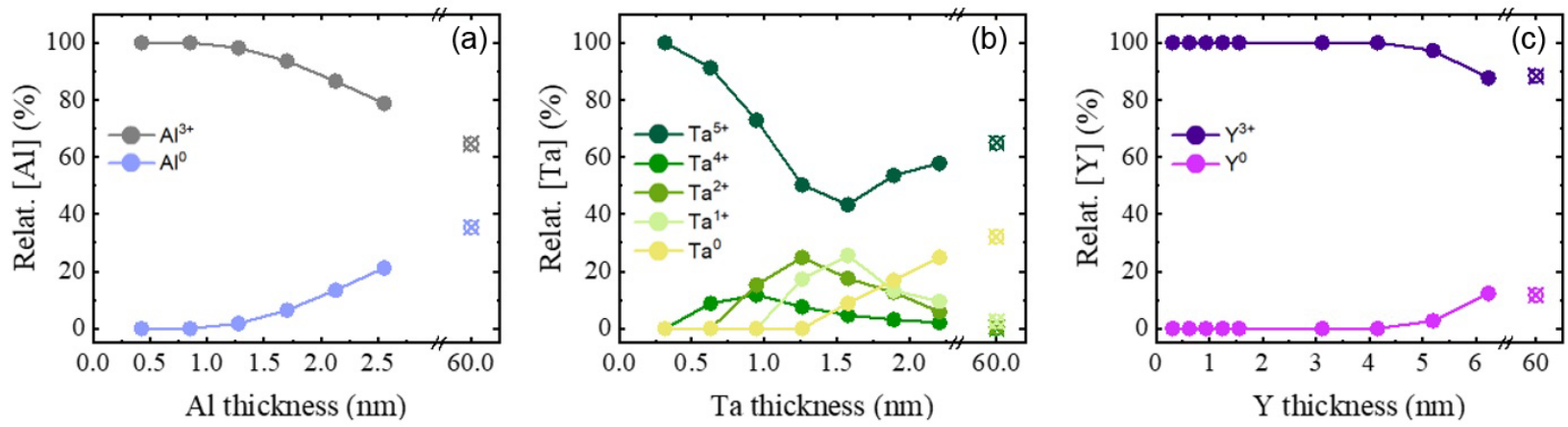

FIG. 5. Relative fractions of oxide and metals for aluminium (a), tantalum (b), and yttrium (c) as a function of the metal thickness. 

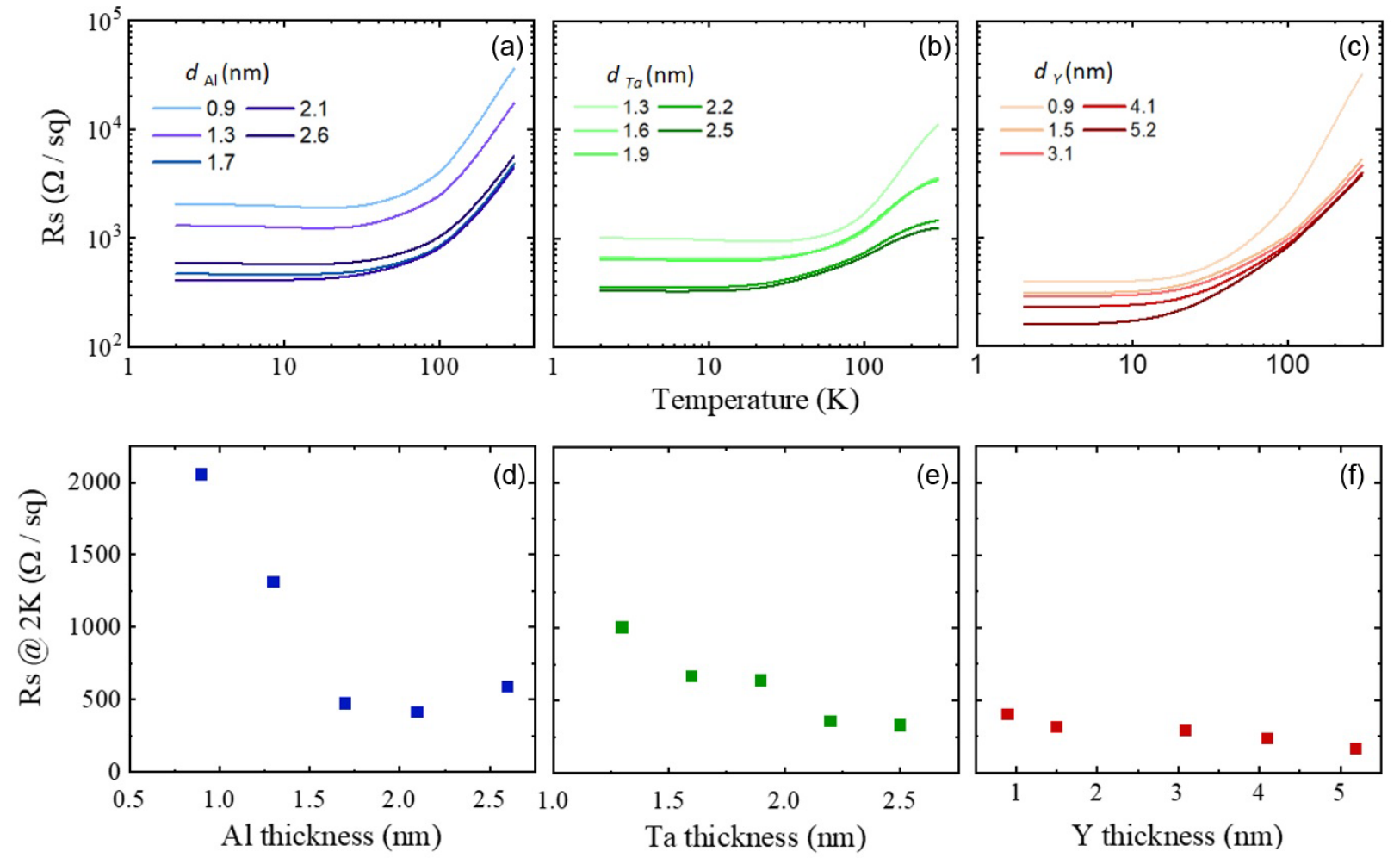

FIG. 6. Top row: Temperature dependence of the sheet resistance of the samples when varying the thickness of the aluminium (a), tantalum (b), and yttrium (c). Bottom row: Plots for the sheet resistance at $T=2 \mathrm{~K}$ by varying the thickness of the aluminium (d), tantalum (e), and yttrium (f).

Figures 7(a)-7(c) show the Hall resistance curves at $2 \mathrm{~K}$ for each system and for different metal thicknesses. The carrier densities and mobilities of the different 2DEGs have been extracted by fitting the nonlinear Hall data [Figs. 7(a)-7(c)] using a twoband model and the sheet resistance at 2 K [Figs. 6(d)-6(f)]. A general trend is that the carrier density increases with the deposited metal thickness until it saturates, for different values depending on the metal: $n_{\mathrm{Al}} \approx 9 \times 10^{13} \mathrm{~cm}^{-2}$, $n_{\mathrm{Ta}} 7 \times 10^{13} \mathrm{~cm}^{-2}, \quad n_{\mathrm{Y}} 4.5 \times 10^{13} \mathrm{~cm}^{-2}$. In addition, we also observe that the carrier density saturates at different thicknesses depending on the metal deposited. This occurs when there is no further reduction of STO by increasing the metal thickness. For $\mathrm{Al}, \mathrm{Ta}$, and $\mathrm{Y}$ the saturation of carrier densities occurs at $1.6,1.2$, and $1.8 \mathrm{~nm}$, respectively. These thicknesses match well with the critical metal thicknesses obtained from XPS, cf. Fig. 3.

By comparing the carrier densities of each system, it can be observed that $\mathrm{Al}$ shows the highest carrier density while Y exhibits the lowest. However, we observed from the XPS data that the reduction of STO was maximum for Y. So, it was expected to have higher carrier densities in Y. Therefore, it has not been possible to correlate the concentration of $\mathrm{Ti}^{3+}$ into the STO with the transport properties of the 2DEG present at the interface between the different oxides. As reflected in this study, a higher concentration of oxygen vacancies in STO does not necessarily lead to higher densities of mobile carriers. The formation of 2DEG are complex processes where the ionic and electronic transport through the oxide layer seems to be the determinant factor in the final properties of the 2DEG.
Also, the role of metal-induced gap states, defects, and fixed charges must be considered.

\section{Transport measurements on $\mathrm{NiFe} / \mathrm{metal} / \mathrm{STO}$ samples}

To perform spin pumping experiments, we prepared samples in which an ultrathin metal layer $(0.9-1 \mathrm{~nm}$ of $\mathrm{Al}, \mathrm{Ta}$, or Y on a STO substrate) was covered by a NiFe thin film, and then capped with $\mathrm{Al}$ (that fully oxidizes when the samples are taken out of the chamber and prevents oxidation of the NiFe). Two sets of samples were prepared with either $2 \mathrm{~nm}$ (for transport experiments) or $20 \mathrm{~nm}$ (for spin-pumping experiments) of $\mathrm{NiFe}$. The results for samples with $0.9-1 \mathrm{~nm}$ of metal are shown in Table II. The carrier concentration increases when going from $\mathrm{Ta}$ to $\mathrm{Al}$ and to $\mathrm{Y}$. The trend is consistent with that of the $\mathrm{Ti}^{3+}$ fraction measured in XPS (extracted from Fig. 3), which indicates that the $\mathrm{NiFe}$ prevents the reoxidation of the STO after exposure to air.

TABLE II. Relative fraction of Ti species from in situ XPS in metal//STO samples with 0.9-1-nm-thick metal layer and 2DEG carrier density extracted from Hall data for $\mathrm{NiFe}(2 \mathrm{~nm}) / \operatorname{metal}(0.9-1$ $\mathrm{nm}) / /$ STO samples.

\begin{tabular}{lcccc}
\hline \hline Metal & $\mathrm{Ti}^{2+}(\%)$ & $\mathrm{Ti}^{3+}(\%)$ & $\mathrm{Ti}^{4+}(\%)$ & $n_{\mathrm{tot}}\left(\mathrm{cm}^{-2}\right)$ \\
\hline $\mathrm{Ta}$ & 9.5 & 18.6 & 73.6 & $6.66 \times 10^{13}$ \\
$\mathrm{Al}$ & 6.1 & 24.6 & 69.3 & $9.22 \times 10^{13}$ \\
$\mathrm{Y}$ & 6.1 & 29.8 & 64.1 & $10.5 \times 10^{13}$ \\
\hline \hline
\end{tabular}



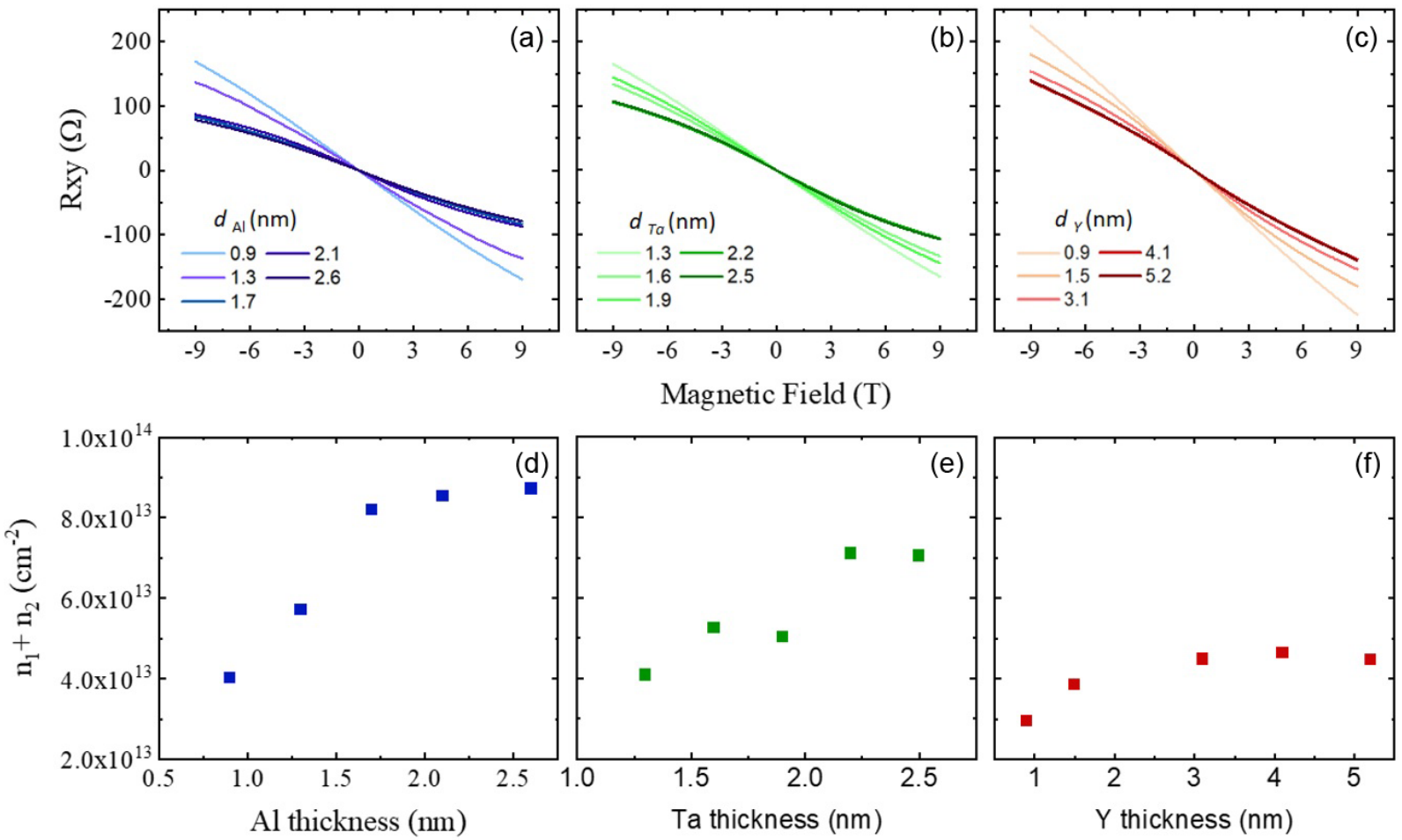

FIG. 7. Hall resistance curves for $\mathrm{Al}$ (a), Ta (b), and $\mathrm{Y}$ (c) and carrier densities of the $2 \mathrm{DEG}$ at $T=2 \mathrm{~K}$ for $\mathrm{Al}$ (d), $\mathrm{Ta}$ (e), and $\mathrm{Y}$ (f) as a function of metal deposited thickness.

\section{Spin pumping experiments in $\mathrm{NiFe} / \mathrm{metal} / \mathrm{STO}$ samples}

Figure 8 presents the results of the SP-FMR experiments on three $\mathrm{NiFe} / \mathrm{metal} / \mathrm{STO}$ samples with 1 -nm-thick metal layers. At the ferromagnetic resonance, a pure spin current is injected from the NiFe into the STO 2DEG with a spin polarization parallel to the in-plane magnetization. The data shown in Fig. 8 correspond to the transverse voltage generated when ferromagnetic resonance is reached. Angle-dependent
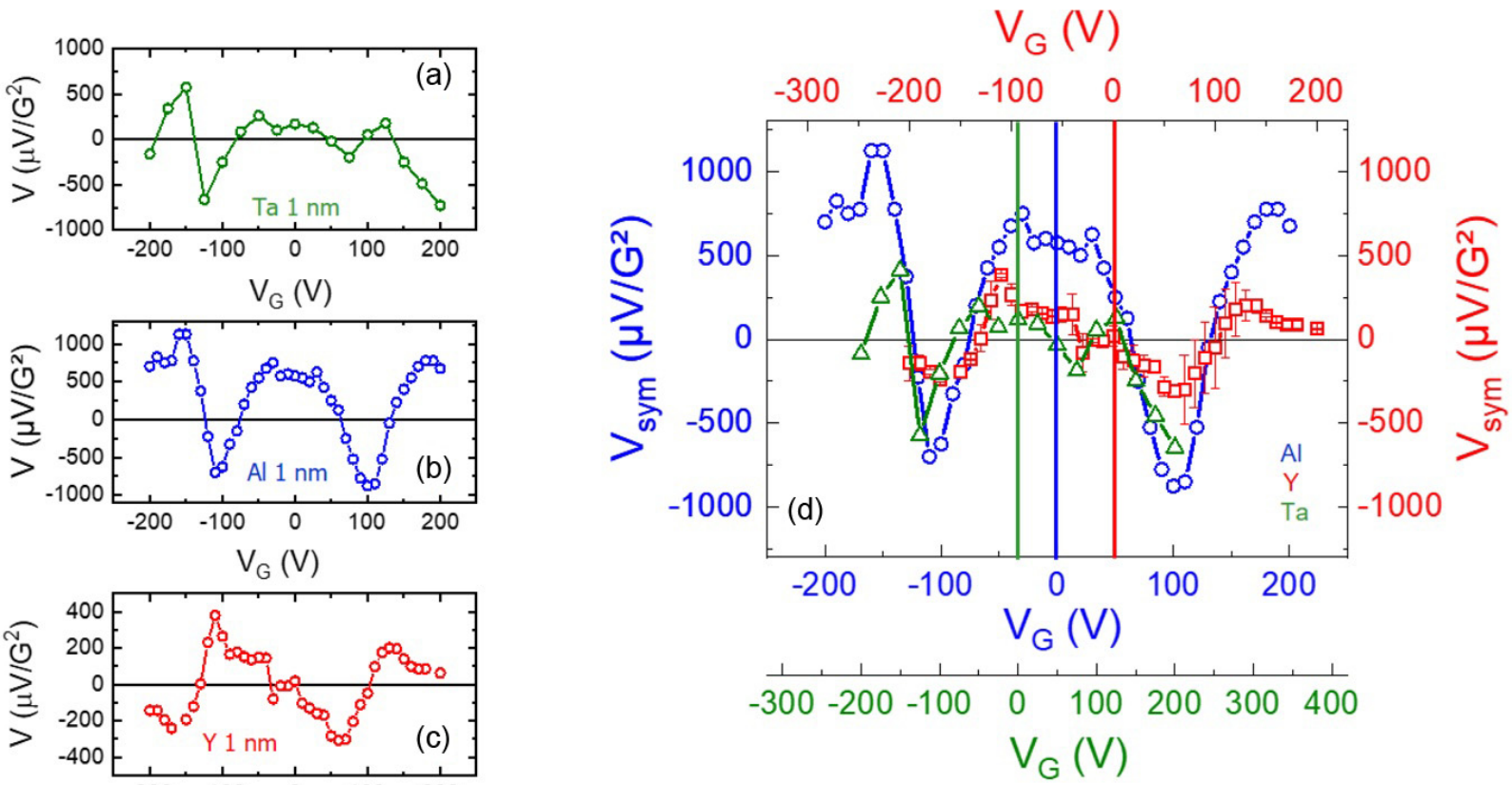

FIG. 8. Gate dependence of the transverse voltage measured during SP-FMR experiments for NiFe $(20 \mathrm{~nm}) / \mathrm{metal}(1 \mathrm{~nm}) / / \mathrm{STO}$ samples for Ta (a), Al (b), and Y (c). (d) Same data replotted by shifting and/or expanding the gate voltage axis for better comparison (see text for details). The vertical lines indicate the zero gate position for the three systems. The data for $\mathrm{Al}$ are replotted from Ref. [11]. 
measurements (not shown here) discard spin rectification effects and attribute the measured signal to spin charge conversion occurring at the Rashba $2 \mathrm{DEG}$ due to the inverse Edelstein effect [24,32]. Figures 8(a)-8(c) display this signal as a function of the back gate voltage and the data present a number of similarities. First, for all three systems the measured voltage is large, corresponding to signals one to two orders of magnitude higher than what is typically found with, e.g., Pt or Ta. This is consistent with previous results in STO 2DEGs that showed remarkably high spin-charge conversion efficiencies $[11,14,15,33,34]$. The figure of merit $\left(\lambda_{\text {IEE }}\right)$ of the spin-charge conversion efficiencies is defined as the ratio between the detected two-dimensional charge current and the injected three-dimensional spin current, and is expected to be proportional to the system's Rashba coefficient and the momentum relaxation time, i.e., $\lambda_{\text {IEE }}=\alpha_{R} \tau / \hbar$. STO 2DEGs exhibit values of $\lambda_{\text {IEE }}$ in excess of $10 \mathrm{~nm}$, i.e., higher than in other Rashba systems [13] or topological insulators [35]. Second, the signal strongly varies with the gate voltage, showing several sign changes with negative and positive maxima. This control of the spin-charge conversion constitutes an additional degree of freedom with respect to the spin-orbitronics stacks based on the spin Hall effect. In an earlier paper combining SP-FMR data with angle-resolved photoelectron spectroscopy and theoretical calculations, we showed that these features can be related to the complex multiorbital band structure of STO 2DEGs [11]. The observed maxima correspond either to the onset of conversion in bands having opposite signs for their spin-charge conversion efficiencies or to specific avoided crossing points, some of which have a topological character $[14,36]$.

The maxima occur at slightly different gate voltage values for Ta, Al, and Y samples, which reflects slight differences in the position of the Fermi energy across the band structure as carriers are accumulated or depleted into the 2DEG by the gate voltage. This is expected since the carrier densities in the ungated case are different for each metal, cf. Table II. Also, the difference in gate voltage positions between the two negative maxima varies between samples, being around 200 $\mathrm{V}$ for $\mathrm{Al}$ and $\mathrm{Y}$ but closer to $300 \mathrm{~V}$ for Ta. This may reflect different efficiencies in the electrostatic gating, for instance, due to different built-in electric fields in the 2DEG and/or different dielectric constant values.

Figure 8(d) replots the data from Figs. 8(a)-8(c) with the same vertical scale but with the horizontal axes slightly shifted and/or stretched to match the position of the two negative maxima. The shifts are consistent with a Fermi level position at zero gate voltage being lower for Ta than for $\mathrm{Al}$ and for $\mathrm{Y}$ - that is, with an ungated carrier density lower for Ta than for $\mathrm{Al}$ and for $\mathrm{Y}$, in line with the results of Table II.

While the main features of the spin-charge conversion efficiency as a function of gate voltage can be explained by band structure effects and subtle variations in the carrier density between samples, the absolute amplitude of the conversion differs between Al/STO, Ta/STO, and Y/STO 2DEGs. This is a priori surprising if the various maxima observed in the gate dependencies correspond to the same points in the band structure for all three systems. In fact, another ingredient is needed: as mentioned above, the conversion efficiency is proportional to the (effective) Rashba coefficient $\alpha_{\mathrm{R}}$ and to the scattering time $\tau$. $\tau$ is not restricted to the 2DEG only, but must also account for the possibility for electrons to leak out of the 2DEG through the metal oxide-acting as a tunnel barrier-to quickly scatter in the $\mathrm{NiFe}$ (the scattering time in metals is in the fs range, while it is in the ps range in the 2DEG). In fact, Al, Ta, and Y oxide tunnel barriers have different tunneling transparencies, quantified by their resistance area (RA) product, and may thus result in various effective $\tau$ values. We can relate the escape time to the RA product through $\tau_{\mathrm{esc}}=\frac{\mathrm{RA} e^{2} m^{*}}{2 \pi \hbar^{2}}$ with $e$ the electron charge and $m^{*}$ the effective mass, and thus calculate the effective scattering time $\tau_{\text {eff }}=\left(\tau_{2 \mathrm{DEG}}{ }^{-1}+\tau_{\mathrm{esc}}{ }^{-1}\right)^{-1}$ with $\tau_{2 \mathrm{DEG}}$ the scattering time in the 2DEG. While $\sim 1-n m$-thick $\mathrm{AlO}_{\mathrm{x}}$ and $\mathrm{TaO}_{\mathrm{x}}$ barriers have relatively high RA values, in the order of $1 \mathrm{M} \Omega \mu \mathrm{m}^{2}$, corresponding to $\tau_{\text {esc }}$ in the ns range or longer, $\mathrm{YO}_{\mathrm{x}}$ barriers are less resistive with RA values closer to $1 \mathrm{k} \Omega \mu \mathrm{m}^{2}$ [37-39], leading to a $\tau_{\text {esc }}$ in the ps range. Since $\tau_{2 \mathrm{DEG}}$ is also in the ps range, significant leakage is expected to occur in the $\mathrm{Y}$ sample, leading to a reduction of the spin-charge conversion efficiency, as observed experimentally.

\section{CONCLUSION}

In summary, we have investigated the generation of 2DEGs in STO by the deposition of various reactive metals (Al, $\mathrm{Ta}, \mathrm{Y}$ ) for a broad range of metal thicknesses. The $2 \mathrm{DEG}$ forms when the metal reacts with the oxygen ions present in the STO, creating oxygen vacancies and releasing electrons. We have monitored this process through XPS and notably associated the appearance of $\mathrm{Ti}^{3+}$ with the population of the $\mathrm{Ti} t_{2 g}$ states. The $\mathrm{Ti}^{3+}$ content increases with the metal thickness deposited, saturating more quickly for $\mathrm{Al}$ and Ta than for Y. The sheet resistance and carrier density decrease and increase, respectively, with the metal thickness for all three systems, which provides a convenient means to design 2DEG with a specific carrier density, something that is hard to control with $\mathrm{LaAlO}_{3} / \mathrm{SrTiO}_{3} 2 \mathrm{DEGs}$. We also combined these 2DEGs with ferromagnetic overlayers used to inject a spin current into the 2DEG and studied the spin-charge conversion process due to the inverse Edelstein effect as a function of the gate voltage. We found very large conversion efficiencies and a complex gate dependence, consistent with earlier results in $\mathrm{LaAlO}_{3} / \mathrm{SrTiO}_{3}$ and Al/STO 2DEGs, confirming that the complex multiorbital band structures drive the spin-charge conversion physics. The tunability of the 2DEG properties, their simple elaboration procedure, and their efficient spin charge conversion capability not only make STO 2DEGs appealing for MESOtype devices but should also motivate experiments to probe further charge-spin conversion and spin-orbit torque. More generally, our results expand the research on STO 2DEGs for spintronics and open avenues towards the design of STO 2DEGs based on other reactive metals displaying functional properties [40-42].

\section{ACKNOWLEDGMENTS}

This work received support from the ERC Advanced Grant No. 833973 "FRESCO," the QUANTERA project "QUANTOX," the Laboratoire d'Excellence LANEF (Grant No. 
ANR-10-LABX-51-01), the ANR project TOPRISE (Grant No. ANR-16-CE24-0017), and Intel's Science and Technol- ogy Center - Feinman. F.T, acknowledges support by Research Grant No. VKR023371 (SPINOX) from Villum Fonden.
[1] A. Ohtomo and H. Y. Hwang, A high-mobility electron gas at the $\mathrm{LaAlO}_{3} / \mathrm{SrTiO}_{3}$ heterointerface, Nature (London) 427, 423 (2004).

[2] S. Gariglio, A. D. Caviglia, J.-M. Triscone, and M. Gabay, A spin-orbit playground: Surfaces and interfaces of transition metal oxides, Rep. Prog. Phys. 82, 012501 (2019).

[3] A. Barthelemy, N. Bergeal, M. Bibes, A. Caviglia, R. Citro, M. Cuoco, A. Kalaboukhov, B. Kalisky, A. Perroni, J. Santamaria, D. Stornaiuolo, and M. Salluzzo, Quasi-Two-dimensional electron gas at the oxide interfaces for topological quantum physics, Europhys. Lett. 133, 17001 (2021).

[4] N. Reyren, S. Thiel, A. D. Caviglia, L. F. Kourkoutis, G. Hammerl, C. Richter, C. W. Schneider, T. Kopp, A.-S. Ruetschi, D. Jaccard, M. Gabay, D. A. Muller, J.-M. Triscone, and J. Mannhart, Superconducting interfaces between insulating oxides, Science 317, 1196 (2007).

[5] A. D. Caviglia, S. Gariglio, N. Reyren, D. Jaccard, T. Schneider, M. Gabay, S. Thiel, G. Hammerl, J. Mannhart, and J.-M. Triscone, Electric field control of the $\mathrm{LaAlO}_{3} / \mathrm{SrTiO}_{3}$ interface ground state, Nature (London) 456, 624 (2008).

[6] A. D. Caviglia, M. Gabay, S. Gariglio, N. Reyren, C. Cancellieri, and J.-M. Triscone, Tunable Rashba Spin-Orbit Interaction at Oxide Interfaces, Phys. Rev. Lett. 104, 126803 (2010).

[7] J. Varignon, L. Vila, A. Barthélémy, and M. Bibes, A new spin for oxide interfaces, Nat. Phys. 14, 322 (2018).

[8] A. Brinkman, M. Huijben, M. van Zalk, J. Huijben, U. Zeitler, J. C. Maan, W. G. van der Wiel, G. Rijnders, D. H. A. Blank, and $\mathrm{H}$. Hilgenkamp, Magnetic effects at the interface between non-magnetic oxides, Nat. Mater. 6, 493 (2007).

[9] R. Jany, C. Richter, C. Woltmann, G. Pfanzelt, B. Förg, M. Rommel, T. Reindl, U. Waizmann, J. Weis, J. A. Mundy, D. A. Muller, H. Boschker, and J. Mannhart, Monolithically integrated circuits from functional oxides, Adv. Mater. Interfaces 1, 1300031 (2014).

[10] P. Irvin, Y. Ma, D. F. Bogorin, C. Cen, C. W. Bark, C. M. Folkman, C.-B. Eom, and J. Levy, Rewritable nanoscale oxide photodetector, Nat. Photonics 4, 849 (2010).

[11] D. C. Vaz, P. Noël, A. Johansson, B. Göbel, F. Y. Bruno, G. Singh, S. McKeown-Walker, F. Trier, L. M. Vicente-Arche, A. Sander, S. Valencia, P. Bruneel, M. Vivek, M. Gabay, N. Bergeal, F. Baumberger, H. Okuno, A. Barthélémy, A. Fert, L. Vila et al., Mapping spin-charge conversion to the band structure in a topological oxide two-dimensional electron gas, Nat. Mater. 18, 1187 (2019).

[12] S. Manipatruni, D. E. Nikonov, C.-C. Lin, T. A. Gosavi, H. Liu, B. Prasad, Y.-L. Huang, E. Bonturim, R. Ramesh, and I. A. Young, Scalable energy-efficient magnetoelectric spin-orbit logic, Nature (London) 565, 35 (2019).

[13] J. C. Rojas Sánchez, L. Vila, G. Desfonds, S. Gambarelli, J. P. Attané, J. M. De Teresa, C. Magén, and A. Fert, Spin-to-charge conversion using rashba coupling at the interface between nonmagnetic materials, Nat. Commun. 4, 2944 (2013).
[14] E. Lesne, Y. Fu, S. Oyarzun, J. C. Rojas-Sánchez, D. C. Vaz, H. Naganuma, G. Sicoli, J.-P. Attané, M. Jamet, E. Jacquet, J.-M. George, A. Barthélémy, H. Jaffrès, A. Fert, M. Bibes, and L. Vila, Highly efficient and tunable spin-to-charge conversion through rashba coupling at oxide interfaces, Nat. Mater. 15, 1261 (2016).

[15] J.-Y. Chauleau, M. Boselli, S. Gariglio, R. Weil, G. de Loubens, J.-M. Triscone, and M. Viret, Efficient spin-to-charge conversion in the $2 \mathrm{D}$ electron liquid at the $\mathrm{LAO} / \mathrm{STO}$ interface, Europhys. Lett. 116, 17006 (2016).

[16] T. C. Rödel, F. Fortuna, S. Sengupta, E. Frantzeskakis, P. L. Fèvre, F. Bertran, B. Mercey, S. Matzen, G. Agnus, T. Maroutian, P. Lecoeur, and A. F. Santander-Syro, Universal fabrication of $2 \mathrm{D}$ electron systems in functional oxides, Adv. Mater. 28, 1976 (2016).

[17] B. Leikert, J. Gabel, M. Schmitt, M. Stübinger, P. Scheiderer, L. Veyrat, T.-L. Lee, M. Sing, and R. Claessen, Controlling the electronic interface properties of $\mathrm{AlO}_{x} / \mathrm{SrTiO}_{3}$ heterostructures, Phys. Rev. Mater. 5, 065003 (2021).

[18] D. C. Vaz, E. Lesne, A. Sander, H. Naganuma, E. Jacquet, J. Santamaria, A. Barthélémy, and M. Bibes, Tuning up or down the critical thickness in $\mathrm{LaAlO}_{3} / \mathrm{SrTiO}_{3}$ through in situ deposition of metal overlayers, Adv. Mater. 29, 1700486 (2017).

[19] R. A. Robie, B. S. Hemingway, and J. R. Fisher, Thermodynamic Properties of Minerals and Related Substances at 298.15 K and 1 Bar (105 Pascals) Pressure and at Higher Temperatures, U.S. Geological Survey (USGPO, Washington DC, 1978).

[20] C. T. Campbell, Ultrathin metal films and particles on oxide surfaces: Structural, electronic and chemisorptive properties, Surf. Sci. Rep. 27, 1 (1997).

[21] Q. Fu and T. Wagner, Interaction of nanostructured metal overlayers with oxide surfaces, Surf. Sci. Rep. 62, 431 (2007).

[22] E. Lesne, N. Reyren, D. Doennig, R. Mattana, H. Jaffrès, V. Cros, F. Petroff, F. Choueikani, P. Ohresser, R. Pentcheva, A. Barthélémy, and M. Bibes, Suppression of the critical thickness threshold for conductivity at the $\mathrm{LaAlO}_{3} / \mathrm{SrTiO}_{3}$ interface, Nat. Commun. 5, 4291 (2014).

[23] J. Millman, A useful network theorem, Proc. IRE 28, 413 (1940).

[24] P. Noël, M. Cosset-Cheneau, V. Haspot, V. Maurel, C. Lombard, M. Bibes, A. Barthelemy, L. Vila, and J.-P. Attané, Negligible thermal contributions to the spin pumping signal in ferromagnetic metal-platinum bilayers, J. Appl. Phys. 127, 163907 (2020).

[25] M. Murata, K. Wakino, and S. Ikeda, X-ray photoelectron spectroscopic study of perovskite titanates and related compounds: An example of the effect of polarization on chemical shifts, J. Electron. Spectrosc. Relat. Phenom. 6, 459 (1975).

[26] M. Sing, G. Berner, K. Goß, A. Müller, A. Ruff, A. Wetscherek, S. Thiel, J. Mannhart, S. A. Pauli, C. W. Schneider, P. R. Willmott, M. Gorgoi, F. Schäfers, and R. Claessen, Profiling the Interface Electron Gas of $\mathrm{LaAlO}_{3} / \mathrm{SrTiO}_{3}$ Heterostructures 
with Hard X-Ray Photoelectron Spectroscopy, Phys. Rev. Lett. 102, 176805 (2009).

[27] S. A. Chambers, M. H. Engelhard, V. Shutthanandan, Z. Zhu, T. C. Droubay, L. Qiao, P. V. Sushko, T. Feng, H. D. Lee, T. Gustafsson, E. Garfunkel, A. B. Shah, J.-M. Zuo, and Q. M. Ramasse, Instability, intermixing and electronic structure at the epitaxial $\mathrm{LaAlO}_{3} / \mathrm{SrTiO}_{3}(001)$ heterojunction, Surf. Sci. Rep. 65, 317 (2010).

[28] G. Drera, G. Salvinelli, A. Brinkman, M. Huijben, G. Koster, H. Hilgenkamp, G. Rijnders, D. Visentin, and L. Sangaletti, Band offsets and density of $\mathrm{Ti}^{3+}$ states probed by x-ray photoemission on $\mathrm{LaAlO}_{3} / \mathrm{SrTiO}_{3}$ heterointerfaces and their $\mathrm{LaAlO}_{3}$ and $\mathrm{SrTiO}_{3}$ bulk precursors, Phys. Rev. B 87, 075435 (2013).

[29] A. B. Posadas, K. J. Kormondy, W. Guo, P. Ponath, J. GelerKremer, T. Hadamek, and A. A. Demkov, Scavenging of oxygen from $\mathrm{SrTiO}_{3}$ during oxide thin film deposition and the formation of interfacial 2DEGs, J. Appl. Phys. 121, 105302 (2017).

[30] R. Nakamura, T. Toda, S. Tsukui, M. Tane, M. Ishimaru, T. Suzuki, and H. Nakajima, Diffusion of oxygen in amorphous $\mathrm{Al}_{2} \mathrm{O}_{3}, \mathrm{Ta}_{2} \mathrm{O}_{5}$, and $\mathrm{Nb}_{2} \mathrm{O}_{5}$, J. Appl. Phys. 116, 033504 (2014).

[31] Y. Ikuma, Y. Tsubaki, Y. Nakao, Y. Yokogawa, and M. Yoshimura, Oxygen diffusion in the fluorite-related phases of $\mathrm{Y}_{2} \mathrm{O}_{3} \mathrm{Ta}_{2} \mathrm{O}_{5}$ and $\mathrm{Ho}_{2} \mathrm{O}_{3} \mathrm{Ta}_{2} \mathrm{O}_{5}$ systems, Solid State Ionics 4041, 258 (1990).

[32] J.-C. Rojas-Sánchez, M. Cubukcu, A. Jain, C. Vergnaud, C. Portemont, C. Ducruet, A. Barski, A. Marty, L. Vila, J.-P. Attané, E. Augendre, G. Desfonds, S. Gambarelli, H. Jaffrès, J.-M. George, and M. Jamet, Spin pumping and inverse spin hall effect in germanium, Phys. Rev. B 88, 064403 (2013).

[33] S. Ohya, D. Araki, L. D. Anh, S. Kaneta, M. Seki, H. Tabata, and M. Tanaka, Efficient intrinsic spin-to-charge current conversion in an all-epitaxial single-crystal perovskite-oxide heterostructure of $\mathrm{La}_{0.67} \mathrm{Sr}_{0.33} \mathrm{MnO}_{3} / \mathrm{LaAlO}_{3} / \mathrm{SrTiO}_{3}$, Phys. Rev. Research 2, 012014(R) (2020).
[34] F. Trier, D. C. Vaz, P. Bruneel, P. Noel, A. Fert, L. Vila, J.P. Attané, A. Barthélémy, M. Gabay, H. Jaffrès, and M. Bibes, Electric-field control of spin current generation and detection in ferromagnet-free $\mathrm{SrTiO}_{3}$-based nanodevices, Nano Lett. 20, 395 (2019).

[35] J.-C. Rojas-Sánchez, S. Oyarzún, Y. Fu, A. Marty, C. Vergnaud, S. Gambarelli, L. Vila, M. Jamet, Y. Ohtsubo, A. TalebIbrahimi, P. Le Fèvre, F. Bertran, N. Reyren, J.-M. George, and A. Fert, Spin to Charge Conversion at Room Temperature by Spin Pumping into a New Type of Topological Insulator: A -Sn Films, Phys. Rev. Lett. 116, 096602 (2016).

[36] M. Vivek, M. O. Goerbig, and M. Gabay, Topological states at the (001) surface of $\mathrm{SrTiO}_{3}$, Phys. Rev. B 95, 165117 (2017).

[37] T. Dimopoulos, G. Gieres, S. Colis, J. Wecker, Y. Luo, and K. Samwer, Magnetic tunnel junctions with yttrium oxide barrier, Appl. Phys. Lett. 83, 3338 (2003).

[38] T. Dimopoulos, G. Gieres, S. Colis, R. Lopez, M. Vieth, J. Wecker, Y. Luo, and K. Samwer, Tunnel junctions with yttrium oxide barrier and various ferromagnetic electrodes, IEEE Trans. Magn. 40, 2296 (2004).

[39] K. Komatsu, S. Kasai, S.-L. Li, S. Nakaharai, N. Mitoma, M. Yamamoto, and K. Tsukagoshi, Spin injection and detection in a graphene lateral spin valve using an yttrium-oxide tunneling barrier, Appl. Phys. Express 7, 085101 (2014).

[40] P. Noël, F. Trier, L. M. Vicente Arche, J. Bréhin, D. C. Vaz, V. Garcia, S. Fusil, A. Barthélémy, L. Vila, M. Bibes, and J.-P. Attané, Non-volatile electric control of spin-charge conversion in a $\mathrm{SrTiO}_{3}$ rashba system, Nature (London) 580, 483 (2020).

[41] J. Bréhin, F. Trier, L. M. Vicente-Arche, P. Hemme, P. Noël, M. Cosset-Chéneau, J.-P. Attané, L. Vila, A. Sander, Y. Gallais, A. Sacuto, B. Dkhil, V. Garcia, S. Fusil, A. Barthélémy, M. Cazayous, and M. Bibes, Switchable two-dimensional electron gas based on ferroelectric $\mathrm{Ca}: \mathrm{SrTiO}_{3}$, Phys. Rev. Mater. 4, 041002(R) (2020).

[42] P. Lömker, T. C. Rödel, T. Gerber, F. Fortuna, E. Frantzeskakis, P. Le Fèvre, F. Bertran, M. Müller, and A. F. Santander-Syro, Two-dimensional electron system at the magnetically tunable $\mathrm{EuO} / \mathrm{SrTiO}_{3}$ interface, Phys. Rev. Mater. 1, 062001(R) (2017). 